Short Communication

\section{A Time: Philosophy of Science}

\author{
Berov G Lyubomir*
}

Engineer, Independent Innovative Ideas Researcher, Smolyan 4700, Bulgaria

\section{Abstract}

Dear reader, isn't it time to turn things around and allow TIME to take its rightful place? In a nutshell, I believe Time should be considered as the primary energy that has created the whole material world and governs the continuous changes in it. In my article "An Intelligent Cosmos: Philosophy of Science", I discuss that this primary creative energy is, more generally, the Dark Energy in our Universe. I believe that Dark Energy probably has a variety of specific manifestations, and I assume that one of those manifestations is Time.

This article is a continuation of my article "An Intelligent Cosmos: Philosophy of Science"
Dear reader, have you ever asked yourself the question "What is Time?" The most basic answer is that time is what we measure with a clock. But people have always liked to clarify things as rationally as possible. Some of the smartest and most erudite amongst us have reached a comprehensive answer to the question "What is Time?" Their answer is simple: "There is no Time."

The other thing that we humans love so much is, justified or unjustified, contradicting each other. And this is how, I think, the truth gets born! And I hope it is indeed true, because in this article I hypothetically assume, in contradiction to the general belief, that time not only exists, but it also is that powerful intelligent force, or rather intelligent energy field, that has created our material world and continues to govern the changes in it. In accordance with my article about the Intelligent Cosmos, referenced here, I assume Time is one of the manifestations of the Dark Energy.

\section{So: What is Time?}

We can trace the search for an answer as far back as the time of St. Augustine. He believed that Time exists only in our consciousness and depends only on how we interpret it. The ancient philosopher explained that people can say that something lasts long or short, but there is no real way to objectively assess Time. So, St. Augustine reached the logical conclusion that the concept of Time exists exclusively in our heads. Well, we all understands this to some degree, don't we? Remarkably, an answer to the question about the essence of Time has been offered by all of the great philosophers, physicists and psychologists in human history, since ancient times to nowadays. And there has never been a single answer.
Time is both subjective and objective. There may be time,

\begin{abstract}
More Information
*Address for Correspondence: Berov G Lyubomir, Engineer, Independent Innovative Ideas Researcher, Smolyan 4700, Bulgaria, Email: my_kaly1@mbox.contact.bg

Submitted: August 14, 2020

Approved: April 21, 2021

Published: April 22, 2021

How to cite this article: Lyubomir BG

A Time: Philosophy of Science. Int J Phys Res Appl. 2021; 4: 031-032.

DOI: 10.29328/journal.ijpra.1001038

Copyright: @ 2021 Lyubomir BG. This is an open access article distributed under the Creative Commons Attribution License, which permits unrestricted use, distribution, and reproduction in any medium, provided the original work is properly cited.
\end{abstract}

Keywords: Time; General theory of relativity; Quantum theory; gravity; Absolute time

Check for updates

OPEN ACCESS but there may not be. The notion that Time is a physical quantity where material changes occur is often replaced by a more convenient one, where Time is simply the order of changes in the material world.

Let's look at things in more detail. According to Sir Isaak Newton, time is absolute, independent, and moves at a constant speed. However, since Newton, the interpretation of Time has been constantly changing. The most significant changes occurred with the development of Einstein's General Theory of Relativity (GTR).

Generally, Einstein's interpretation of Time is: "Time has no independent existence except in the order of events by which we measure it. Time is a sequence of events - that is my conclusion." In the process of creating the GTR, Einstein struggled to decipher the physical phenomenon of gravity. In 1912 he had a genius inspiration. He realized that massive cosmic bodies, such as planets, stars, galaxies, and black holes, distort time, and this distortion is the cause of gravity. He expressed this idea in mathematical formulas, but its essence can be described in the following manner: "Everything strives to exist where it ages the most slowly. The physical result of this strive is the gravitational pull."

And the longer the time delay, the stronger the gravitational pull. On Earth, the time delay is only a few microseconds a day, so the force of Earth gravity is weak. On the surface of a neutron star, where the time delay is several hours a day, the gravitational pull is extremely strong. And near the surface of 
a black hole, where time is almost stopped, the gravitational pull is so powerful that even light cannot escape it.

But in parallel with Einstein's work, other great physicists developed two new branches of physics. The first is quantum physics - a field that tries to clarify the behavior of the smallest particles in the universe. The second is the theory of the corpuscular-wave dualism. This theory argues that the world manifests itself as both waves and particles. And so, problems arose. In both theories, the concept of time does not exist at all in the most fundamental level of matter.

For many years, physicists have tried to unite the GTR with the other two fields of physics by creating the Great Unifying Equation, assuming that everything in the universe must be interconnected, from the smallest particles to the vast galaxies. Just over 40 years ago, two genius physicists, John Wheeler and Bryce-De Witt, almost developed such a theory. However, their theory turned out to be controversial because, at its core, the concept of time did not exist. And the deeper the physical science went into world of atoms, protons, quarks, etc., the less relevant the concept of time became.

Dear reader, isn't it time to turn things around and allow TIME to take its rightful place? In a nutshell, I believe Time should be considered as the primary energy that has created the whole material world and governs the continuous changes in it. In my article,, An Intelligent Cosmos: Philosophy of Science", I discuss that this primary creative energy is, more generally, the Dark Energy in our Universe. I believe that Dark Energy probably has a variety of specific manifestations, and I assume that one of those manifestations is Time.

Below I present my particular hypothesis on Time:

1. Is Time an energy field?

We have already seen earlier how the gravitational field and Time are inextricably linked. Typically, in physics such inseparable connections exist only between the same type of phenomena. As such, I believe that just as gravity is an energy field, so is time. Next, if, according to my theory, time is the primary intelligent energy field that has created our universe, then time must have created a material object, which in turn generates the gravitational field. This is how time has created, and continues to create our material world. And if Time has also created the dark matter, then we can say that Time is the other name of the Dark Energy. The ideas above are still very schematic, but at the moment we do not need to go any deeper.

2. Why in the field of atoms, protons, quarks, etc. time does not appear?

Probably because in this field the values of the various physical quantities related to time are so small that, at least for now, they are elusive to us, both theoretically and empirically.

\section{Why is Time an intelligent energy field?}

The view that Time is a sequence of events is shared by most scientists in the modern world. But a sequence of events that provides a stable world that has been around for billions of years is clearly linked to an unimaginably powerful intellect because only the creatures of the intellect are long-lasting. And, in the case of superior intellects, such as the Dark Energy, and by association Time as one of its manifestations, they are practically eternal. At least we don't see the end of the world for now, do we?

Dear reader, I hope that this short article gives you a clearer picture of the world we live in. I hope I'm right.

\section{References}

1. Brian G. The Hidden Reality. 2011.

2. Richard B. The Physics of Miracles. 2009.

3. Carlos C. The Art of Dreaming. 1993.

4. Hawking SW. A Brief History of Time. 1973.

5. Vera R. Bright Galaxies, Dark Matter. 1997 